\title{
Innovación al sistema de tutorías para fortalecer su uso e impacto en la acción tutorial
}

\author{
Innovation to the tutoring system to strengthen its use and impact in the tutorial \\ action
}

RODRÍGUEZ-RAMÍREZ, Felipe†*, GARCÍA-ORTEGA, Irene y VÁSQUEZ-GAMBOA, Saira Antonieta

Tecnológico Nacional de México - Instituto Tecnológico de Tehuacán, México.

ID $1^{\mathrm{er}}$ Autor: Felipe, Rodríguez-Ramírez / ORC ID: 0000-0002-1991-3390, CVU CONACYT ID: 899136

ID $1^{\text {er }}$ Coautor: Irene, García-Ortega / ORC ID: 0000-0001-9638-7577, CVU CONACYT ID: 997104

ID $2^{\text {do }}$ Coautor: Saira Antonieta, Vásquez-Gamboa / ORC ID: 0000-0001-7031-0467, CVU CONACYT ID: 997110

DOI: $10.35429 / \mathrm{JCP} .2020 .11 .4 .9 .17$

Recibido 15 de Enero, 2020; Aceptado 30 de Junio, 2020

\section{Resumen}

La tutoría ha sido implementada en las instituciones de educación superior como una estrategia que permita contribuir al mejoramiento del desempeño académico de los estudiantes y coadyuvar a su formación integral, favoreciendo la eficiencia terminal en las instituciones. En el Instituto Tecnológico de Tehuacán se desarrolló un sistema web con el objetivo de ser una herramienta de apoyo en el desarrollo de la acción tutorial. Este sistema se puso en funcionamiento en el año 2019, para analizar el impacto de su puesta en funcionamiento. El presente trabajo presenta los resultados de la aplicación del sistema y se presenta el desarrollo de una innovación al sistema, utilizando un framework de código abierto, compatible con la base de datos, que se desarrolló con el objetivo de contar con un sistema que integre a cada uno de los actores del programa de tutorías, que les ayude a desarrollar la acción tutorial de una manera más sencilla y productiva al integrar mejoras y nuevas funcionalidades $y$ en consecuencia con el apoyo del sistema lograr favorecer al cumplimiento de los objetivos del programa. El análisis se desarrolló bajo una metodología cualitativa y el desarrollo del sistema bajo una metodología ágil.

Tutoría, Innovación, Acción tutorial

\begin{abstract}
Tutoring has been implemented in higher education institutions as a strategy that contributes to the improvement of the students' academic performance as well as their comprehensive training while improving efficiency in the institutions. At the Instituto Tecnológico de Tehuacán, a web system was developed with the aim of being a support tool in the development of the Tutoring initiatives. This system was operated in 2019 , to analyze the impact of its implementation. This document presents the results of the system implementation as well as its innovative approach, which consists of using an opensource framework, compatible with a database that was developed with the objective of having a system that integrates the main actors from the tutoring program, and helps them develop tutoring initiatives in a simpler and more productive way by integrating improvements and new features. Therefore, the system will impact in successfully achieving the goals of the tutoring program. The analysis was executed under a qualitative methodology and the development of the system under an agile methodology.
\end{abstract}

Tutoring, Innovation, Tutorial action

Citación: RODRÍGUEZ-RAMÍREZ, Felipe, GARCÍA-ORTEGA, Irene y VÁSQUEZ-GAMBOA, Saira Antonieta. Innovación al sistema de tutorías para fortalecer su uso e impacto en la acción tutorial. Revista de Pedagogía Crítica. 2020. 411:9-17.

\footnotetext{
* Correspondencia al Autor (Correo electrónico: feliperodriguezramirez@ittehuacan.edu.mx)

$\uparrow$ Investigador contribuyendo como primer autor.
} 


\section{Introducción}

La tutoría es una estrategia que ha sido implementada desde hace tiempo en las instituciones educativas con el propósito de fortalecer la formación integral de los estudiantes y apoyar en problemas como son: el rezago, reprobación, deserción y baja eficiencia terminal (ANUIES, 2018), dada su importancia esta estrategia sigue vigente, ha dado lugar a diversas investigaciones, diversas propuestas y acciones encaminadas a fortalecer su aplicación y al logro de los objetivos para los cuales fue presentada. Actualmente y con los avances tecnológicos existen investigaciones con propuestas relacionadas con el uso de la tecnología en la acción tutorial por mencionar algunas se encuentran las siguientes: "el uso de herramientas tecnológicas en la acción tutorial, mejora la función docente, permitiendo abarcar mayor número de situaciones con posibilidades de éxito, ofreciendo una mayor individualización, y aumentando la eficiencia en el trabajo" (Torres, 2013). Por otro lado, en otra investigación se menciona la importancia de aplicar las nuevas tendencias tecnológicas, en el ámbito tutorial para contribuir con el quehacer académico, obtener más productividad, mejores resultados, fortalecer la comunicación y aprovechamiento del tiempo (Ramírez, Alcuida, López, Rodríguez, \& Santana, 2015).

La tutoría en los Institutos Tecnológicos se ha implementado como una estrategia de apoyo para la formación integral del alumno, donde el docente se convierte en su acompañante a través de su proceso de formación profesional, desarrollando en su quehacer tutorial diversas actividades que tienen el propósito de estimular el desarrollo humano integral del estudiante.

En busca de implementar estrategias en favor de la tutoría se desarrolló un sistema web para apoyar a los tutores en su acción tutorial, este sistema beneficia a todos los actores del programa de tutorías ya que automatiza muchas de las tareas que cada uno realiza, sin embargo uno de los desafíos que se presenta es el cambio que representa para las personas que llevan varios años desarrollando sus actividades de manera manual, y a partir de la presentación del sistema se les presenta el reto de aprender a utilizarlo, lo cual requiere tiempo para capacitarse, dando lugar a un periodo de capacitación con muchos retos.
Después de un semestre en el que se dio capacitación a las diferentes áreas que participaron, se realizó un análisis FODA (Fortalezas, Oportunidades, Debilidades y Amenazas), para evaluar la efectividad del sistema web, lo cual dio lugar a desarrollar una innovación al sistema que está funcionando actualmente pero que integra mejoras, así como nuevas funcionalidades en beneficio de la acción tutorial y en consecuencia favorecer al logro de los objetivos de la tutoría. Para aplicar el análisis FODA se parte de cuál fue el objetivo de desarrollar un Sistema web para la gestión del programa de tutorías en el Instituto Tecnológico de Tehuacán, y a través de la aplicación de instrumentos, así como de la técnica de la observación ver hasta qué punto el sistema web está cumpliendo con el objetivo, que factores están impulsando su cumplimiento y cuáles podrían dañarlo o indicar mejoras para el sistema.

Es importante la investigación desarrollada ya que permite elaborar propuestas que beneficien a los estudiantes del Instituto Tecnológico de Tehuacán al brindarles la oportunidad de que el proceso de acompañamiento sea más objetivo, más cercano ya que proporciona una herramienta de comunicación constante entre tutores y tutorados, que no requiere de la presencia física de ambos actores en un aula o espacio para tal fin. Por otro lado, a los tutores les facilita el trabajo de planear su acción tutorial, al presentar información con aspectos relevantes obtenidos a partir de la aplicación de los anexos del manual del tutor, lo que le permite realizar diagnósticos para brindar a los tutorados una atención más oportuna a sus necesidades de atención tutorial y con esto un apoyo más efectivo en su formación integral.

Con esta propuesta se beneficia también al logro de las metas institucionales del Instituto Tecnológico de Tehuacán y servirá como base para poder ser implementado en otros Institutos Tecnológicos. Este artículo se encuentra integrado de la siguiente manera:

Planteamiento del problema: aquí se presenta el planteamiento del problema que da la apertura a la realización de la investigación, el objetivo general y la pregunta de investigación. 
Metodología: en este apartado se presenta la metodología utilizada.

Desarrollo: en esta sección se presenta la descripción de las actividades realizadas.

Resultados: aquí se presenta el sistema web con la utilización de un framework en su desarrollo.

Conclusiones: se presentan las conclusiones del trabajo.

Agradecimientos: en este espacio se presentan los agradecimientos a las diferentes instancias y personas que apoyaron en la realización del proyecto.

\section{Planteamiento del problema}

En el año 2019 se implementó en el Instituto Tecnológico de Tehuacán un sistema web que apoya la acción tutorial e integra a los diferentes actores del programa de tutorías. Se dio un periodo de capacitación a las diferentes áreas académicas donde se muestra el funcionamiento del sistema, y su relación con el programa de tutorías. En la Figura 1 se presenta la capacitación que se dio a los tutores del departamento de Económico Administrativas donde se encuentran tutores de las carreras de Ingeniería en Gestión Empresarial, Contador Público y Licenciatura en Administración.

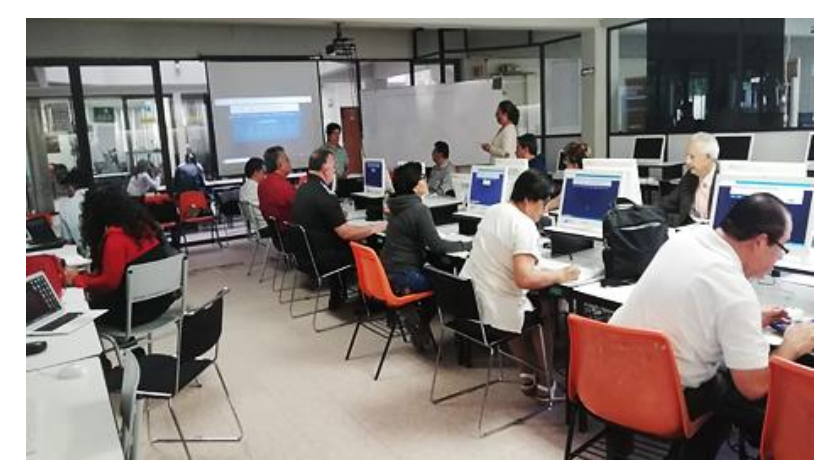

Figura 1 Capacitación de tutores del Departamento Económico Administrativas

Fuente: (https://www.facebook.com/institutotecnologicot ehuacan.itteh/)
El reto principal en este periodo de capacitación, fue la resistencia inicial que se da al presentar una alternativa que cambia la manera en la que llevan su actividad, sin embargo resalta la falta de capacitación de los docentes en su rol de tutores, ya que la mayoría realiza actividades diferentes, aisladas lejos de atender necesidades de atención tutorial de los tutorados lo cual es un factor importante que repercute en la baja efectividad del programa de tutorías, al conocer el sistema y darse cuenta de las diferentes actividades que pueden realizar dependiendo de las necesidades de atención que presentan sus tutorados, y las bondades del sistema, se muestra interés en muchos de los tutores en conocer el sistema para aprovechar las ventajas que les presenta, sin embargo algunos lo vieron complejo y decidieron seguir trabajando a su manera su acción tutorial. Considerando el interés y la importancia que presenta el sistema como una herramienta de apoyo, se realiza un análisis FODA del sistema web que permita evaluar la efectividad del sistema e identificar mejoras que permitan favorecer el impacto en el uso del sistema.

\section{Objetivo General}

Innovar el Sistema Integral para la gestión del Programa de tutorías, utilizando un framework, para mejorar los procesos que se incluyen en el programa de tutorías que se utiliza en el Instituto Tecnológico de Tehuacán.

\section{Pregunta de investigación}

¿Analizar la efectividad del sistema de tutorías favorecerá la implementación de nuevas funcionalidades y mejoras al sistema?

\section{Metodología}

Para el desarrollo de la investigación se utilizó una metodología cualitativa, la cual se interesa por captar la realidad social a través de los ojos de la gente que está siendo estudiada" (Monje, 2011). Por otro lado, el método a seguir fue el de investigación-acción ya que no solo se desea conocer una realidad determinada de un grupo, sino también se desea intervenir para resolver una problemática, este método puede llevarse a cabo por grupos de personas que realizan una actividad colectiva en bien de todos, para establecer cambios apropiados a una situación determinada (Colmenares E. \& Piñeiro M., 2008). 
El método de investigación-acción utiliza un modelo en espiral de ciclos de planificación, acción, observación y reflexión.

Se utiliza este método ya que es el que se sigue desde el inicio de la investigación donde el resultado fue la propuesta del sistema web, se reflexiona ahora en relación a la efectividad del sistema a través de un análisis FODA y se planifica una propuesta de innovación que integre nuevas funcionalidades y mejoras que impacten en el uso del sistema y en consecuencia en la efectividad del programa de tutorías en el Instituto Tecnológico de Tehuacán.

Para el desarrollo del proyecto se siguieron las siguientes etapas:

Aplicar instrumentos de investigación para la recolección de información que permita realizar el análisis FODA del sistema web.

Analizar la información obtenida, para determinar las nuevas funcionalidades y mejoras que se integraran al sistema.

Desarrollar la innovación al sistema con la ayuda de un framework de código abierto compatible con la base de datos.

Para el desarrollo de la innovación se utilizó una metodología ágil, dado que se parte de un sistema que tiene bien establecidos los procesos que va a integrar, lo que se necesita ahora es una metodología que permita el desarrollo de software de manera ágil respondiendo a los cambios que pudieran surgir durante el desarrollo del proyecto.

\section{Desarrollo}

Un análisis FODA es una herramienta muy utilizada para identificar "cuáles son los recursos con los que cuento que me dan la posibilidad de llegar a mi objetivo, y por otra parte cuál es el impacto de las debilidades, así como de las amenazas y oportunidades que el medio ofrece" (García López \& Cano Flores, s.f.). Por otro lado se menciona que al crear un análisis FODA, se verá de manera más clara hasta qué punto el sitio web está cumpliendo con los objetivos, cuáles son los factores que están impulsando este cumplimiento, cuales son factores para el éxito y cuáles podrían dañarlo e indicar mejoras para el sitio web (Hostname, 2016).
Para llevar a cabo el análisis FODA, se partió de reflexionar en los siguientes aspectos, en primer lugar ¿cuál es el objetivo del sistema para el cual fue desarrollado?, ¿qué se quiere que los usuarios realicen con el sistema? ¿qué se busca que los usuarios logren con el sistema? ¿qué tiene el sistema web que no tenga otra aplicación que se haya estado utilizado para las tutorías?

Una vez considerando de donde se parte, se procede a el análisis FODA, donde se inicia con la enumeración de las fortalezas internas del sistema.

Para recabar esta información las técnicas que se aplicaron fueron la observación, durante el periodo de capacitación y seguimiento que se dio en el año 2019, la cual se llevó a cabo de manera presencial en el laboratorio de cómputo a cada uno de los actores del sistema: coordinadora institucional de tutorías, jefes de departamento, coordinadores de tutorías de cada una de las áreas académicas, tutores, y tutorados.

Esta manera de distribuir a las personas por roles permitió obtener la información necesaria para lo que se necesitaba. también se utilizó la entrevista donde se pudo recabar información directamente de las personas, una vez que se les había dado la capacitación y en el periodo de seguimiento para indagar acerca de la información que se necesitaba.

Durante el periodo enero-junio 2019 se dio capacitación a todas las áreas académicas del Instituto Tecnológico de Tehuacán, iniciando con la coordinadora institucional, donde se le presentó el módulo al rol que tiene asignado, y las actividades automatizadas en las que está involucrada, su aceptación y el manejo del sistema fue muy rápido, no se manifiesta dificultad en su entendimiento.

Durante la etapa de seguimiento, la coordinadora institucional, manifiesta la necesidad de nuevas funcionalidades en el sistema, que son importantes para el desarrollo de sus actividades y para la implementación del programa de tutorías. 
Por otro lado, se dio capacitación al $100 \%$ de los jefes de departamento, mostrando de manera personalizada la realización de actividades que tienen asignadas los jefes de departamento dentro del sistema, como son la designación del coordinador de tutorías del área, la creación de grupos y la asignación de alumnos tutorados. Este proceso fue muy sencillo, no se les dificultó la realización de estas actividades de tal forma que en el sistema quedaron registrados el $100 \%$ de jefes de departamento, el $100 \%$ de tutores asignados en el periodo enero- junio 2019, y el $100 \%$ de alumnos asignados a tutoría de $1^{\circ}$ y $2^{\circ}$ semestre, como se muestra en la Tabla 1.

\begin{tabular}{|c|c|c|c|}
\hline \multirow[t]{2}{*}{ Área } & \multirow[t]{2}{*}{ No. de tutores } & \multicolumn{2}{|c|}{$\begin{array}{l}\text { No. de alumnos } \\
\text { registrados }\end{array}$} \\
\hline & & $1^{\circ} \mathrm{Sem}$. & $2^{\circ}$ Sem. \\
\hline Ing. Bioquímica & 5 & 32 & 148 \\
\hline Ing. Civil & 2 & 53 & 0 \\
\hline Ing. en Gestión empresarial & 3 & 0 & 38 \\
\hline Ing. Industrial & 5 & 39 & 179 \\
\hline Ing. Logística & 1 & 0 & 47 \\
\hline Ing. Mecatrónica & 7 & 25 & 155 \\
\hline Ingeniería en Sistemas & 9 & 101 & 11 \\
\hline Lic. en Administración & 6 & 30 & 71 \\
\hline Lic. en Contador Público & 8 & 25 & 136 \\
\hline Ing. Electrónica & 7 & 43 & 0 \\
\hline Total & 51 & 318 & 785 \\
\hline
\end{tabular}

Tabla 1 Registro en el sistema de tutores

Fuente: (García Ortega, Vásquez Gamboa, \& Rodríguez Ramírez, 2019)

Después de utilizar el sistema se analizó el impacto que había tenido en su uso por parte de los tutores de todas las áreas académicas a los cuales ya se les había registrado como tutores. Para realizar el análisis se observó quienes dieron seguimiento al uso del sistema hasta terminar el programa de tutorías con sus grupos asignados en el semestre y quienes desistieron de su utilización, este análisis se presenta en la Tabla 2 .

\begin{tabular}{|l|r|r|}
\hline \multicolumn{1}{|c}{ Área } & $\begin{array}{c}\text { Tutor } \\
\text { Nocentes } \\
\text { docentes } \\
\text { registrados }\end{array}$ & $\begin{array}{c}\text { Cuantos } \\
\text { culminaron } \\
\text { el proceso }\end{array}$ \\
\hline Ing. Bioquímica & 5 & 0 \\
\hline Ing. Civil & 2 & 0 \\
\hline Ing. en Gestión empresarial & 3 & 3 \\
\hline Ing. Industrial & 5 & 2 \\
\hline Ing. Logística & 1 & 0 \\
\hline Ing. Mecatrónica & 7 & 1 \\
\hline Ingeniería en Sistemas & 9 & 9 \\
\hline Lic. Administración & 6 & 6 \\
\hline Lic. en Contador Público & 8 & 7 \\
\hline Ing. Electrónica & 7 & 0 \\
\hline Total & 53 & 28 \\
\hline
\end{tabular}

Tabla 2 Tutores registrados en el sistema de tutorías, y los que terminaron el proceso

Fuente (García Ortega, Vásquez Gamboa, \& Rodríguez. Ramírez, 2019)
En la tabla 2 se observa que el $47 \%$ de los tutores que se registraron no culminaron el proceso de tutorías.

Una vez concluido este periodo de capacitación y seguimiento se procedió a realizar el análisis FODA del sistema obteniendo los resultados siguientes.

\section{Fortalezas internas del sistema}

Para realizar este listado de fortalezas, se muestran las fortalezas con las que cuenta el sistema actual que no están presentes en el sistema que se utilizaba como apoyo al programa de tutorías anteriormente. Estas fortalezas son:

Sistema $100 \%$ enfocado en apoyar la tutoría integrando a cada uno de los actores del programa de tutorías.

Sistema con perfiles y funcionalidades para cada uno de los actores del programa.

Sistema que permite la comunicación bidireccional entre tutores y tutorados.

- $\quad$ Sistema que permite elaborar diagnósticos para la atención de necesidades de tutoría de los tutorados.

- Sistema que automatiza las actividades realizadas por cada uno de los actores del programa de tutorías.

Sistema que automatiza los anexos de apoyo al tutor del manual del tutor del TecNM.

- $\quad$ Sistema con un diseño responsive.

Debilidades internas.

- $\quad$ Diseño web que no utiliza framework.

- $\quad$ Algunas funcionalidades confusas.

- $\quad$ Sistema de navegación, confuso, basado en menús.

- $\quad$ Diseño que no es $100 \%$ responsive.

- Duplicidad de información. 
Oportunidades externas.

Disponibilidad de frameworks de código abierto, que utilizan el mismo patrón de diseño MVC (Modelo, Vista, Controlador) del sistema y cuentan con funcionalidades ya creadas que pueden ser utilizadas para centrarse en lo que se quiere resolver, desarrollar o mejorar.

\section{Usuarios que ya conocen la estructura del} sistema.

Usuarios muy interesados en utilizar el sistema y aprovechar las ventajas que les ofrece en el desarrollo de su actividad tutorial.

Oportunidades de capacitación a los docentes en cuanto a la formación de tutores por parte del Tecnológico Nacional de México, a través de la disposición del diplomado en formación de tutores lo que ayuda a que los usuarios estén más conscientes del compromiso que se tiene al ser tutores.

- Mayor acercamiento al uso de la tecnología al tener que hacer uso de ella, ante esta situación emergente que se está viviendo en el país y en mundo.

\section{Amenazas externas.}

- Desinterés por algunos usuarios al resistirse a un cambio en el desarrollo de actividad tutorial y al uso del sistema.

- No obligatoriedad para que los docentes participen en la formación de tutores.

- Desinterés para su implantación en el Tecnológico.

Una vez que se analizó esta información se procedió a realizar las modificaciones necesarias en la base de datos con la finalidad de darle mayor estabilidad al sistema, mejorar procesos y agregar nuevas funcionalidades. Dentro de los cambios principales se encuentra el manejo de catálogos lo cual permitirá mejor y más eficiente manejo de la información, por otro lado, al revisar las tablas se eliminaron algunas que contenían información duplicada y también se les aplicaron reglas de normalización.
En la etapa de analizar que Framework se podía utilizar se partió de la idea de utilizar un Framework para innovar el sistema actual ya que esta realizado en su totalidad en código PHP, y un framework actualmente ofrece muchas ventajas, se han convertido en herramientas muy utilizadas por desarrolladores web, los cuales al contar con librerías y/o diversas funcionalidades, les permite centrarse en lo que desea realizar o resolver al poder utilizar estas funcionalidades que normalmente son de uso común y que ya están diseñadas. Una definición de lo que es un framework menciona lo siguiente: "un framework es una aplicación reutilizable, semicompleta que puede ser especializada para producir aplicaciones concretas y específicas" (Delía, s.f.).

El utilizar un framework, permite tener un mayor orden y control al incorporar una estructura definida, cuando se instala para PHP. Contiene utilidades para las arquitecturas de desarrollo MVC, niveles de control de acceso, manejo de formularios y validación de usuario lo cual da mayor seguridad, estabilidad $\mathrm{y}$ escalabilidad al sistema.

Se decidió utilizar Laravel el cual es un Framework de código abierto, flexible y robusto que permite crear páginas web de una forma ágil y rápida es compatible con la base de datos del sistema que actualmente se tiene.

\section{Resultados}

Como resultado del análisis y el desarrollo de la innovación se tiene un sistema basado en un framework que presenta, mejoras al sistema que actualmente se está utilizando, así como nuevas funcionalidades que ayudarán a tener un sistema que servirá como apoyo al programa de tutorías con el objetivo de ser más efectivo que el sistema anterior que favorezca en el logro de los objetivos de la tutoría.

En la Figura 2, se muestra la ventana de inicio que fue uno de los cambios realizados, anteriormente el usuario debía elegir su rol de la lista de todos los roles en el sistema e introducir su contraseña, ahora solo ingresa un usuario y su contraseña. 


\section{Ingresar}

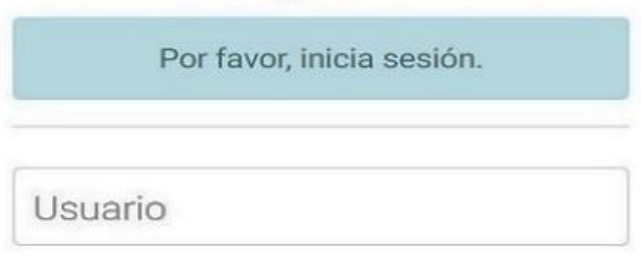

\section{Contraseña}

Mantener la sesión iniciada

INICIAR SESIÓN

Figura 2 Ventana de inicio al Sistema de Tutorías Fuente: Elaboración propia

Si al ingresar un usuario tenía diferentes roles, por ejemplo, si es coordinador de tutorías del área académica y al mismo tiempo es tutor, tenía que acceder eligiendo su rol y si desea cambiar tenía que salir del sistema y nuevamente volver a ingresar, actualmente en la ventana de login, puede ingresar con su usuario y contraseña asignado, la diferencia es que si tiene más de un rol entonces se le presenta una ventana donde puede estar intercambiando de rol sin necesidad de salir del sistema, como se muestra en la Figura 3.

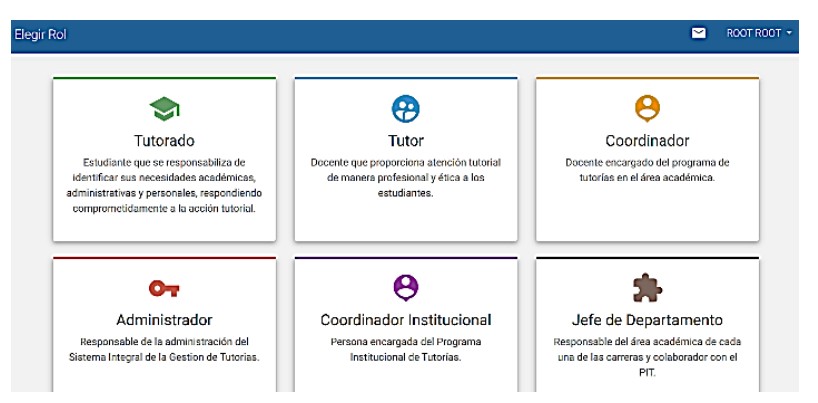

Figura 3 Ventana con todos los roles asignados al usuario root

Fuente: Elaboración propia

Otro de los cambios relevantes en el sistema es la forma de navegación en las ventanas, por ejemplo, uno de los perfiles con más cambios es el correspondiente al perfil tutor, donde la forma de navegación es compleja en el sistema anterior, actualmente al entrar en este rol se le presenta al tutor la ventana de navegación que se muestra en la Figura 4.

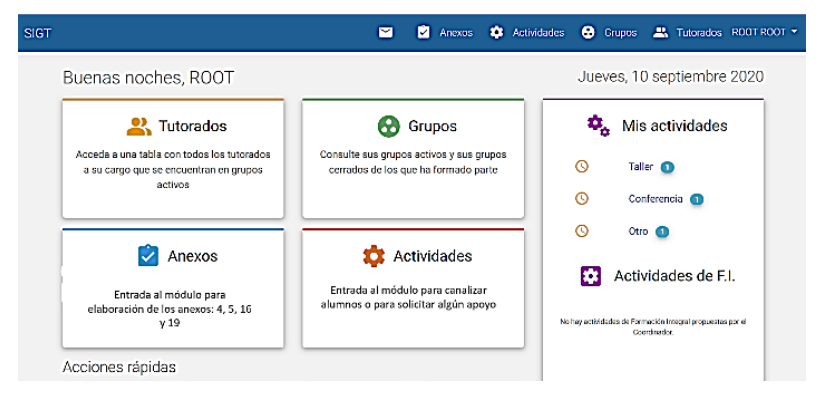

Figura 4 Ventana de navegación en el perfil tutor Fuente: Elaboración propia

En esta ventana el tutor tiene a la mano un espacio donde al ingresar estará observando si tiene mensajes de sus tutorados, podrá acceder a sus grupos ya sean grupos activos o cerrados, o ir directamente a su grupo actual de tutorados.

En el sistema anterior las opciones eran por menús lo cual hacía más difícil la navegación y generaba confusión entre los usuarios, la ventana del sistema anterior se muestra en la Figura 5.

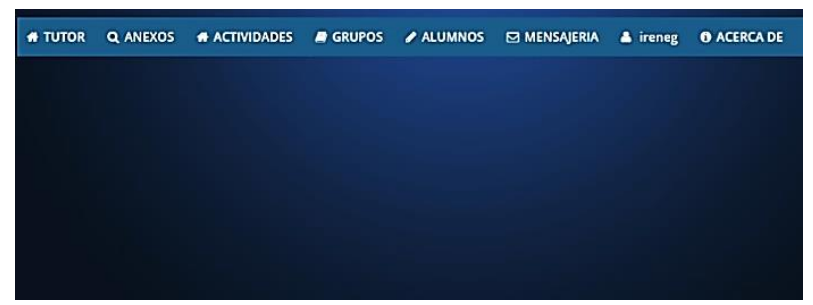

Figura 5 Módulo Tutor del sistema de tutorías anterior Fuente (García Ortega, Vásquez Gamboa, \& Rodríguez Ramírez, 2019)

Como se observa en la Figura 5 el tutor debe navegar a través de un sistema de menús que genera en los usuarios confusión al tener que grabarse en que opción se encuentra la ventana que quiere acceder. Por ejemplo, en la mejora al sistema, si desea ir a su listado de alumnos en su ventana simplemente va a elegir alumnos tutorados y en esta ventana tendrá toda la información de sus tutorados como se muestra en la Figura 6.

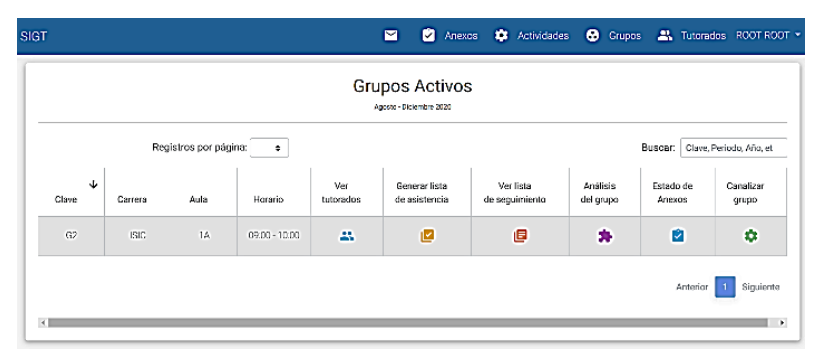

Figura 6 Ventana con la información de los grupos del tutor

Fuente: Elaboración propia 
Como se observa en la Figura 6, el tutor tendrá a la mano toda la información de sus alumnos tutorados de una manera más agradable a la vista con resultados que le permitan poder canalizar o solicitar algún servicio para atender alguna problemática detectada en el grupo o de manera individual, además podrá regresar a la opción del menú principal que desee sin tener que regresar ventana por ventana como en el sistema anterior, donde además para realizar alguna canalización o para solicitar algún servicio tenía que salir de la ventana que estaba consultando a otro opción del menú principal, lo cual se observó causaba confusión en los tutores.

Estos son los cambios que en general se han realizado al sistema de tutorías que se está utilizando, donde las ventanas son más agradables y al cambiar de un sistema de menús a un sistema con tarjetas hace más fácil la navegación a través de las diferentes opciones con las que cuenta.

Integrando además nuevas funcionalidades que están diseñadas para simplificar las actividades desarrolladas por cada uno de los actores del programa de tutorías con la finalidad de lograr un impacto en los objetivos del programa de tutorías al tener una atención más oportuna y eficiente de las necesidades de atención tutorial de los estudiantes del Instituto Tecnológico de Tehuacán.

\section{Conclusiones}

Al finalizar el desarrollo del sistema podemos concluir que es de gran importancia el realizar evaluaciones a las herramientas que se utilizan reflexionando si éstas cumplen con los objetivos para los que fueron creadas, y que un análisis FODA es efectivamente un instrumento que ayuda a determinar la efectividad de un sistema y sobre todo a la propuesta de mejoras que permitan impactar de manera más efectiva en el programa de tutorías.

\section{Agradecimientos}

Se externa el agradecimiento a las siguientes instancias que hicieron posible la realización del proyecto: "Innovación al Sistema Integral de tutorías utilizando un framework”.
Al Tecnológico Nacional de México por el financiamiento otorgado para el desarrollo del proyecto.

A los directivos en especial a la directora del Instituto Tecnológico de Tehuacán C. M.E. Yeyetzín Sandoval González, por todo su apoyo en la realización de este proyecto.

A la M.P.H. Patricia Huerta Orozco jefa del departamento de Desarrollo Académico por las facilidades y el apoyo brindado para las diferentes actividades realizadas de capacitación y seguimiento.

- A los alumnos que participaron y colaboraron con la realización del proyecto:

- Andrés Domínguez Valiente.

- José Luis Correa Martínez.

- $\quad$ Erick Alberto Hernández Castillo.

\section{Referencias}

Aguilar, N. M. (17 de diciembre de 2011). El paradigma crítico y los aportes de la investigación acción participativa en la transformación de la realidad social: un análisis desde las ciencias sociales. Obtenido de http://institucional.us.es/revistas/cuestiones/21/ art_14.pdf

ANUIES. (17 de octubre de 2018). Para evitar rezago, reprobación y deserción escolar IPN ofrece sus programas tutoriales. Obtenido de http://www.anuies.mx/noticias_ies/para-evitarrezago-reprobacin-y-desercin-escolar-el-ipnfortalece-sus

Colmenares E., A. M., \& Piñeiro M., M. L. (2008). La investigación acción. Una herramienta metodológica heurística para la comprensión y transformación de realidades y prácticas socio-educativas. Obtenido de http://www.redalyc.org/pdf/761/76111892006.p df 
Delía, L. (s.f.). Framework para el Desarrollo Ágil. Obtenido de http://sedici.unlp.edu.ar/bitstream/handle/10915 /4000/Tesis.\%20Framework\%20para\%20el\%2 0desarrollo\%20\%C3\%A1gil\%20de\%20sistema s\%20web.pdf-

PDFA1b.pdf?sequence $=2 \&$ isAllowed $=y$

García López, T., \& Cano Flores, M. (s.f.). El FODA: una técnica para el análisis de problemas en el contexto de la planeación en las organizaciones. Obtenido de https://www.uv.mx/iiesca/files/2013/01/foda19 99-2000.pdf

García Ortega, I., Vásquez Gamboa, S. A., \& Rodríguez Ramírez, F. (junio de 2019). Sistema Integral para la Gestión del Programa de Tutorías en el Instituto Tecnológico de Tehuacán. Obtenido de https://www.ecorfan.org/republicofperu/researc $\mathrm{h} \_$journals/Revista_de_Tecnologia_y_Educacio n/vol3num8/Revista_de_Tecnolog\%C3\%ADa_ y_Educaci\%C3\%B3n_V3_N8_4.pdf

Hostname. (17 de marzo de 2016). Evalúa la efectividad de tu sitio web mediante un análisis FODA. Obtenido de https://www.hostname.cl/blog/evalua-laefectividad-de-tu-sitio-web-mediante-unanalisis-foda

Monje, Á. C. (2011). Metodología de la investigación cuantitativa y cualitativa. Obtenido de https://www.uv.mx/rmipe/files/2017/02/Guiadidactica-metodologia-de-la-investigacion.pdf

Ramírez, P. D., Alcuida, F. E., López, J. A., Rodríguez, R. E., \& Santana, D. E. (2015). Desarrollo de aplicación web para tutorías académicas, incorporando reingeniería de procesos, programación concurrente y sistemas de gestión de bases de datos distribuidas. Revista Iberoamericana de Producción Académica y Gestión Educativa, 9.

TecNM. (Febrero de 2013). Obtenido de MANUAL DEL TUTOR DEL SNIT: http://www.tecnm.mx/images/areas/docencia01 /documentos/MANUAL_DEL_TUTOR.pdf
Torres, G. I. (2 de 04 de 2013). La utilización de las TICS en las tutorías como medio para mejorar las relaciones entre centros-profesorespadres-alumnos.

Obtenido

de https://reunir.unir.net/bitstream/handle/1234567 89/1798/2013_04_04_TFM_ESTUDIO_DEL_ TRABAJO.pdf? sequence $=1$ 